\title{
Notable mixed substrate fermentation by native Kodamaea ohmeri strains isolated from Lagenaria siceraria flowers and ethanol production on paddy straw hydrolysates
}

\author{
Shalley Sharma ${ }^{1}$, Anju Arora ${ }^{1 *}$, Pankhuri Sharma ${ }^{1}$, Surender Singh ${ }^{1}$, Lata Nain ${ }^{1}$ and Debarati Paul ${ }^{2}$
}

\begin{abstract}
Background: Bioethanol obtained by fermenting cellulosic fraction of biomass holds promise for blending in petroleum. Cellulose hydrolysis yields glucose while hemicellulose hydrolysis predominantly yields xylose. Economic feasibility of bioethanol depends on complete utilization of biomass carbohydrates and an efficient co-fermenting organism is a prerequisite. While hexose fermentation capability of Saccharomyces cerevisiae is a boon, however, its inability to ferment pentose is a setback.

Results: Two xylose fermenting Kodamaea ohmeri strains were isolated from Lagenaria siceraria flowers through enrichment on xylose. They showed $61 \%$ glucose fermentation efficiency in fortified medium. Medium engineering with $0.1 \%$ yeast extract and peptone, stimulated co-fermentation potential of both strains yielding maximum ethanol $0.25 \mathrm{~g} \mathrm{~g}^{-1}$ on mixed sugars with $\sim 50 \%$ fermentation efficiency. Strains were tolerant to inhibitors like 5-hydroxymethyl furfural, furfural and acetic acid. Both K. ohmeri strains grew well on biologically pretreated rice straw hydrolysates and produced ethanol.

Conclusions: This is the first report of native Kodamaea sp. exhibiting notable mixed substrate utilization and ethanol fermentation. K. ohmeri strains showed relevant traits like utilizing and co-fermenting mixed sugars, exhibiting excellent growth, inhibitor tolerance, and ethanol production on rice straw hydrolysates.
\end{abstract}

Keywords: Yeast, Kodamaea ohmeri, Fermentation efficiency, Mixed sugar fermentation, Inhibitors, Rice straw hydrolysates

\section{Background}

Recent environmental disturbances, fluctuating prices, and uncertainties associated with the use of conventional fuels, have led to paradigm shift to displace conventional fuels with sustainable, renewable, and environmentally friendly/clean energy sources, among which biomassderived energy appears to be the most promising option [1]. Of various alternative energy sources, bioenergy derived from lignocellulosic biomass has attracted significant attention as one of the routes to address energy

\footnotetext{
*Correspondence: anjudev@yahoo.com

1 Division of Microbiology, ICAR-Indian Agricultural Research Institute, New Delhi 110012, India

Full list of author information is available at the end of the article
}

crisis, especially bioethanol in transport sector [2]. Second generation bioethanol, produced by fermenting sugar slurries obtained from enzymatic hydrolysis of cellulose present in lignocellulosic biomass, has the potential of being a major contributor to meet the global energy demand, as biomass is the most abundant, sustainable, and renewable resource on earth. However, unfavorable economics is the foremost impediment in successful deployment of this process on industrial scale. An efficient pretreatment with lower inhibitor generation followed by enzymatic hydrolysis for maximum sugar recovery, and complete utilization and fermentation of all the sugars present in hydrolysates will aid in making the process cost effective [3]. In addition to cellulose, 
biomass also has hemicellulose, which is the second major polysaccharide, consisting of hexoses and pentoses, with xylose as the major pentose sugar.

Thus, complete conversion of lignocellulosic biomass entails a co-fermenting yeast, capable of fermenting both glucose and xylose yielding high ethanol titers. Development of strains for use in industrial-scale facilities is continuously being carried out in parallel with the process optimization. Commercial strains of $S$. cerevisiae, the most widely used organisms for ethanol production are exclusively involved in glucose fermentation, thus completely utilizing cellulosic fraction while xylose is left unfermented. To overcome this drawback of $S$. cerevisiae, recombinant strains capable of utilizing xylose have been developed since 1980s but ethanol yield was found to be low [4]. Since then, several genetic engineering approaches have been adopted for developing a recombinant strain capable of mixed substrate fermentation but with limited success [5]. This is due to the constraints associated with co-fermentation, like aerobic process of xylose fermentation, co-factor (NADH) imbalance [6] and glucose repression [7, 8]. In addition, inhibitors present in biomass hydrolysates [9] and medium constituents [10] have been observed to affect yeast physiology and fermentation efficiency [11, 12]. All these issues need to be addressed earnestly.

On the other hand, native pentose fermenting yeasts are well known $[4,13]$. First report of ethanol production from xylose by yeast came in 1958 when Karczewska [14] observed ethanol production from Candida tropicalis. Pichia and Scheffersomyces are the most interesting pentose fermenting yeasts but their co-fermenting abilities on mixed substrates are yet to be established to the extent suitable for commercial application [15]. Numerous native yeasts are known for xylose assimilation but very few are reported for efficient fermentation of xylose to ethanol. Such yeast include Pichia, Candida, Pachysolen, Clavispora, Debaromyces, Kluyveromyces, Cryptococcus, Rhodotorula etc. Researchers have demonstrated low to high ethanol production from xylose in rich medium, by different yeasts isolated from natural habitats like tree bark, decaying wood samples and insect gut [16-18]. Mixed substrate utilization and co-fermentation is still a challenge. Thus, rational bio prospecting for native pentose assimilating and fermenting yeasts is the contemporary approach and increasing efforts have recently been put into evaluating natural xylose fermenting potential of yeasts $[19,20]$.

A yeast genus Kodamaea, earlier placed under Pichia genus has been reported for pentose utilization including xylose and arabinose but fermentation of pentoses to ethanol has not been reported. A novel sp. of Kodamaea, K. kakuduensis, isolated from Australian Hibiscus flower, was reported to be a good glucose fermenter with weak xylose assimilation properties [21]. Kodamaea ohmeri has been explored for its food fermentation properties especially for pickling and cocoa beans but ethanol production has not been reported yet [22]. Zhu et al. [23] described D-arabitol as the main product from glucose by K. ohmeri. This study illustrates mixed sugar utilization, ethanol fermentation potential, and inhibitor tolerance of two native $K$. ohmeri strains isolated from the flowers of L. siceraria plant for their possible exploitation in bioethanol production.

\section{Experimental}

\section{Isolation of yeast strains}

Lagenaria siceraria flowers were collected, washed with distilled water and crushed in pestle mortar with $0.8 \%$ saline under aseptic conditions. $1 \mathrm{~mL}$ of this suspension was inoculated into $50 \mathrm{~mL}$ MXYP broth $(0.5 \%$ malt extract, $1 \%$ xylose, $0.5 \%$ yeast extract and $0.3 \%$ peptone, $\mathrm{pH}$ 5) in $100 \mathrm{~mL}$ flasks with $0.25 \%$ sodium propionate, for enrichment of xylose utilizing yeasts. After $48 \mathrm{~h}$ incubation at $30{ }^{\circ} \mathrm{C}$, culture samples were plated on MXYP agar with chloramphenicol $\left(50 \mu \mathrm{g} \mathrm{mL}{ }^{-1}\right)$ antibiotic. Plates were incubated for $24 \mathrm{~h}$ at $30^{\circ} \mathrm{C}$ and colonies were selected based on their morphology. Selected colonies were purified and grown on same medium and glycerol stocks were prepared.

Identification and characterization of selected yeast strains Two potent xylose assimilating strains were selected, strain 5 and strain 6 . Both the strains were characterized on morphological, biochemical as well as on molecular level. Phenotypic characterization was done on the basis of their colony and cell morphology using phase contrast microscopy and scanning electron microscopy. Molecular characterization included sequencing of the ITS region of the yeast strains.

\section{Studying cell morphology using phase contrast microscopy and scanning electron microscopy}

To study morphology, overnight grown cultures were observed under phase contrast microscope (Olympus America Inc.) at magnification $10 \times$ and $40 \times$. Cell morphology was also studied using scanning electron microscope (Zeiss EVOMA10). Overnight incubated cultures on xylose $(1 \mathrm{~mL})$ were centrifuged at $8000 \mathrm{~g}$ for $10 \mathrm{~min}$, $2.5 \%$ glutaraldehyde fixative was added to the pellet and kept for 2-4 h to arrest growth. Cultures were then washed with $0.1 \mathrm{M}$ phosphate buffer thrice at an interval of $15 \mathrm{~min}$. Samples were dehydrated with a graded series of acetone (30, 50, 70, 80, 90, 95 and 100\%), fixed on cover slips placed over stuff grids. A drop of hexamethyl disilazone was added over the cover slips and then 
allowed to dry in a fume hood. Cells were observed with scanning electron microscope at an acceleration voltage of $20 \mathrm{kV}$ and images recorded.

\section{Molecular identification through ITS sequencing}

Further confirmation was done by PCR amplification of ITS region. PCR procedures involved denaturation at $95{ }^{\circ} \mathrm{C}$ for $5 \mathrm{~min}$, followed by 35 cycles of $94{ }^{\circ} \mathrm{C}$ for $5 \mathrm{~min}, 55^{\circ} \mathrm{C}$ for $30 \mathrm{~s}$ and extension at $72{ }^{\circ} \mathrm{C}$ for $45 \mathrm{~s}$, with final extension for $10 \mathrm{~min}$ at $72{ }^{\circ} \mathrm{C}$. Amplified products were run over $1 \%$ agarose gel to confirm their molecular size. ITS sequencing of the amplified products was completed by Xcelris, India and further analyzed using Basic Local Alignment Search Tool (BLAST) [24]. Partial sequencing of the strains was done using ITS 1 and ITS 4 degenerate primers i.e., ITS1-forward primer (5'-TCCGTAGGTGAACCTGCGG-3') and ITS4-reverse primer (5'-TCCTCCGCTTATTGATATGC-3') [25].

\section{Biochemical characterization}

Ability of Kodamaea ohmeri strains to assimilate different sugars was tested using biochemical strips (Hi Media) for yeast. Overnight cultures were inoculated on the strips $(100 \mu \mathrm{L}$ each $)$ and incubated at $28^{\circ} \mathrm{C}$. Results were observed for $72 \mathrm{~h}$.

\section{Determining enzyme activities}

K. ohmeri strains were grown for $48 \mathrm{~h}$ on $2 \%$ xylose, and mixed sugars $(2 \%$ xylose $+2 \%$ glucose $)$ in minimal medium with shaking at $150 \mathrm{rpm}$ at $30{ }^{\circ} \mathrm{C}$. After $48 \mathrm{~h}$, cultures were centrifuged at $8000 \mathrm{rpm}$ for $10 \mathrm{~min}$ and supernatant was discarded. Pellet was processed for xylose reductase (XR) and xylitol dehydrogenase (XDH) activities were measured and expressed as specific activities. Protein concentration in crude extracts was measured using BSA as standard.

\section{Xylose reductase}

Pellet obtained was washed twice with phosphate buffer (250 mM, pH 7.0), sonicated, and the lysate was then used as the crude enzyme extract. Two cocktails were prepared as shown in Table 1 . Crude enzyme was added to the experimental vial $(50 \mu \mathrm{L})$ and readings were taken at $340 \mathrm{~nm}$ for $3 \mathrm{~min}$ and the rate of change of OD was used to determine the activity of the enzyme.

\section{Xylitol dehydrogenase}

Pellet obtained was washed twice with Tris-Cl buffer (500 mM, pH 8.6), sonicated and the lysate was then used as the crude enzyme extract. For this assay, two cocktails were prepared as shown in Table 2, in two separate cuvettes and kept on ice. Crude enzyme $(50 \mu \mathrm{L})$ was added to the experimental vial and measurements
Table 1 Reaction cocktail for xylose reductase activity

\begin{tabular}{lll}
\hline Solution & \multicolumn{2}{l}{ Volume $(\mu \mathrm{L})$} \\
\cline { 2 - 3 } & Control & Experimental \\
\hline DI water & 200 & 100 \\
Potassium phosphate & 600 & 600 \\
2-Mercaptoethanol & 100 & 100 \\
NADPH & 50 & 50 \\
Xylose & 0 & 100 \\
\hline
\end{tabular}

Table 2 Reaction cocktail for xylitol dehydrogenase activity

\begin{tabular}{lll}
\hline Solution & \multicolumn{2}{l}{ Volume $(\mu \mathrm{L})$} \\
\cline { 2 - 3 } & Control & Experimental \\
\hline DI water & 300 & 200 \\
$500 \mathrm{mM}$ tris-HCl & 400 & 400 \\
$2-M e r c a p t o e t h a n o l$ & 100 & 100 \\
$\mathrm{NAD}^{+}$ & 100 & 100 \\
Xylitol & 0 & 100 \\
\hline
\end{tabular}

of the rate of change of absorbance per min at $340 \mathrm{~nm}$ was measured and considered as the $\mathrm{XDH}$ activity for $K$. ohmeri strain 5 and strain 6.

\section{Fermentation abilities of $K$. ohmeri strains}

Both strains were grown in minimal medium (1.36 mg L ${ }^{-1} \mathrm{KH}_{2} \mathrm{PO}_{4}, 0.2 \mathrm{~g} \mathrm{~L}^{-1} \mathrm{MgSO}_{4} \cdot 7 \mathrm{H}_{2} \mathrm{O}, 2.0 \mathrm{~g} \mathrm{~L}^{-1}$ $\mathrm{NaCl}, 1.0 \mathrm{~g} \mathrm{~L}^{-1}\left(\mathrm{NH}_{4}\right)_{2} \mathrm{SO}_{4}, 10 \mathrm{mg} \mathrm{L}^{-1} \mathrm{FeSO}_{4}, \mathrm{pH}$ 5) with $5 \%$ xylose $/ 10 \%$ glucose or both as carbon source for $72 \mathrm{~h}$ at $30{ }^{\circ} \mathrm{C}$ to check their ability to grow and ferment xylose. Effect of salts like $\mathrm{NaCl}$ and $\mathrm{FeSO}_{4}$ was studied. Medium $(50 \mathrm{~mL})$ in $100 \mathrm{~mL}$ Erlenmeyer flasks was inoculated (10\% inoculum) and incubated for $72 \mathrm{~h}$ at $30{ }^{\circ} \mathrm{C}$. Inoculum was prepared in MXYP broth ( $\mathrm{pH} 7.0$ ) by incubating it at $30{ }^{\circ} \mathrm{C}$ for $48 \mathrm{~h}$ and shaking $(150 \mathrm{rpm})$. Aliquots were aseptically withdrawn at regular intervals and the absorbance read at $660 \mathrm{~nm}$ (Specord 200) to measure growth. These aliquots were then centrifuged at 10,000 rpm for $10 \mathrm{~min}$ and supernatants were used for estimation of sugar consumption and ethanol production by HPLC as described later.

\section{Fermentation of mixed sugars}

Cultures were grown on mixed sugars ( $5 \%$ glucose $+5 \%$ xylose $)$ as carbon source in minimal medium $\left(10 \mathrm{~g} \mathrm{~L}^{-1}\right.$ $\mathrm{KH}_{2} \mathrm{PO}_{4}, 5 \mathrm{~g} \mathrm{~L}^{-1}\left(\mathrm{NH}_{4}\right)_{2} \mathrm{SO}_{4}, 5 \mathrm{~g} \mathrm{~L}^{-1} \mathrm{MgSO}_{4} \cdot 7 \mathrm{H}_{2} \mathrm{O}$, $1 \mathrm{~g} \mathrm{~L}^{-1}$ yeast extract, $\mathrm{pH} 5$ ). Composition of minimal medium in this case differed from the above experiment as effect of salts and yeast extract was being monitored. 
Fermentation was carried out in two phases. Incubation at $30{ }^{\circ} \mathrm{C}$ under shaking for $48 \mathrm{~h}$ for biomass production was switched to static conditions for ethanol production. Samples were analyzed for growth, sugar consumption and ethanol production. Fermentation efficiency was calculated as [26].

$$
\begin{aligned}
\% \text { Fermentation Efficiency }= & (\text { Actual Ethanol Yield in grams } \\
& / \text { Theoretical Ethanol Yield in grams }) \\
& \times 100
\end{aligned}
$$

Theoretical Ethanol yield $=$ (sugar consumed in grams

$$
\times 0.511)
$$

\section{Stimulation of fermentation ability upon medium supplementation}

Effect of medium supplementation with yeast extract and peptone on ethanol production was studied. Treatments with combinations of yeast extract $(0.1-1 \%)$ and peptone $(0.1$ and $1 \%)$ with pure or mixed sugars $(10 \%$ glucose or $10 \%$ glucose $+5 \%$ xylose) were applied. Incubation was carried out as described earlier and samples were analyzed for growth and fermentation.

\section{Analytical methods}

Ethanol levels were estimated using chromatographic techniques, such as HPLC and GC.

\section{High performance liquid chromatography}

Cultures were harvested at regular intervals, centrifuged at $8000 \mathrm{rpm}$ for $10 \mathrm{~min}$, filtered using $0.22 \mu$ syringe filters and subjected to analysis by HPLC. Samples were run on Aminex HPX-87H column (Bio-Rad, Hercules, CA, USA) at $65^{\circ} \mathrm{C}$ using $5 \mathrm{mM} \mathrm{H}_{2} \mathrm{SO}_{4}$ as mobile phase at

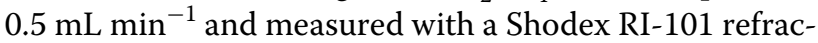
tion index detector (Shoko Scientific Co. Ltd., Yokohama, Japan). Ethanol concentration and sugar consumption were determined.

\section{Inhibitor tolerance of $K$. ohmeri strains}

For exploitation of $K$. ohmeri strains for fermentation of biomass hydrolysates, it is important to check their capability to grow in presence of HMF, furfural, formic acid and acetic acid, the predominant by-products of biomass pretreatment which are present in hydrolysates and reported to inhibit growth.

Cultures were grown in presence of HMF (0.5$\left.5.0 \mathrm{~g} \mathrm{~L}^{-1}\right)$ and furfural $\left(0.25-0.65 \mathrm{~g} \mathrm{~L}^{-1}\right)$ in minimal medium with $5 \%$ glucose $+2.5 \%$ xylose and $0.1 \%$ yeast extract for $96 \mathrm{~h}$. Growth was checked every $24 \mathrm{~h}$ by reading absorbance at $660 \mathrm{~nm}$. Appropriate controls were maintained and growth was compared. Similar experiment was carried out using acetic acid (5-15 $\left.\mathrm{g} \mathrm{L}^{-1}\right)$ and formic acid (3-11 $\left.\mathrm{g} \mathrm{L}^{-1}\right)$ under similar conditions. All the experiments were carried out in triplicates.

\section{Growth and fermentation on biologically pretreated paddy straw hydrolysates}

Rice straw of the aromatic rice (Pusa 2511) was pretreated under solid state fermentation using Trametes hirsute, for 7 days and cellulose content was analysed in pretreated solids [27]. Enzymatic hydrolysis of biologically pretreated solids was carried out using accellerase ${ }^{\circledR} 1500$ (Genencor) loading corresponding to $0.5 \mathrm{~mL}(\sim 15 \mathrm{FPU})$ per g glucan [28]. Total sugars in hydrolysates were estimated using DNS [29].

Both strains were grown in hydrolysates [30] and culture samples were periodically withdrawn. Samples were processed. Growth and sugar consumption were observed. Ethanol production was detected by HPLC. Defined medium with $1.3 \%$ glucose served as control.

Statistical analyses of the results was done using SPSS (Version 21.0. Armonk, NY: IBM Corp).

\section{Results and discussion}

Growth and characterization

Lagenaria siceraria flowers are rich in pentose and hexose sugars and thus used as a source for isolating pentose assimilating K. ohmeri strains [31]. K. ohmeri strains were isolated and purified from $L$. siceraria flowers by enrichment on MXYP medium and maintained as glycerol stocks. Both the strains grew well on minimal medium with xylose as sole carbon source (Additional file 1: Figure S1). They showed distinct opaque, butyroid, creamy, circular colony morphology with regular margins and raised elevation. Under phase contrast microscope, cells appeared ovoid and occurred singly (Additional file 1: Figure S2). Scanning electron microscopy images showed shrunk cells with irregular margins indicating stress. Budding cells were also observed under scanning microscopy (Fig. 1).

Biochemical characterization showed that both strains could assimilate maltose, sucrose, galactose, cellobiose, raffinose, trehalose, glucose and xylose while inositol, dulcitol, lactose, melibiose were not assimilated and urease test was also negative (Additional file 1: Table S1). Both strains were identified to be $K$. ohmeri upon partial sequencing. Strain 5 (GenBank Accession No. KT598022) showed $100 \%$ similarity with K. ohmeri while strain 6 (GenBank Accession No. KT598023) displayed 97\% similarity. Phylogenetic tree constructed using Maximum-Likelihood [32] also showed their relationship with K. ohmeri (Fig. 2). Kodamaea genus was earlier placed under Pichia genus but was separated later due to considerable genetic distances as measured by partial sequences of $18 \mathrm{~S}$ and $26 \mathrm{~S}$ ribosomal RNA and only seven 

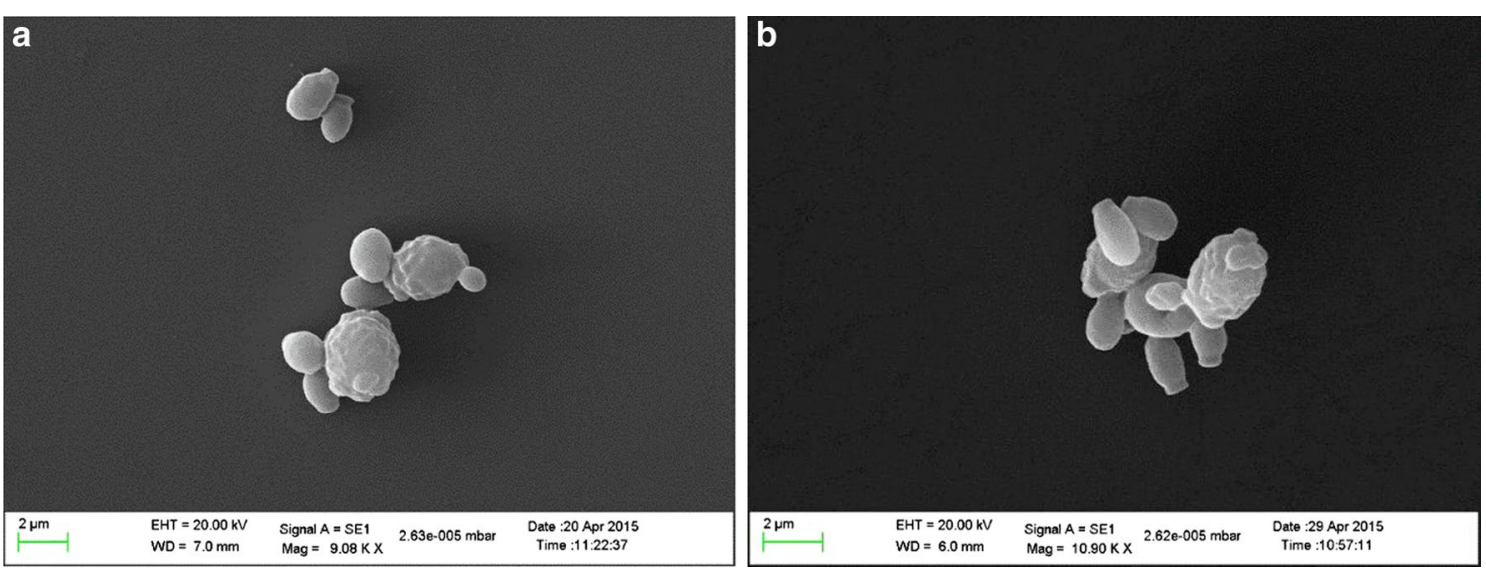

Fig. 1 Scanning electron micrographs of strain 5 and strain 6. Cells of strain 5 (a) and strain 6 (b) appear stressed due to growth on xylose under micro-aerophilic conditions. Budding cells are clearly visible in the electron micrographs

species were placed under the genus Kodamaea including K. anthophila, K. kakaduensis, K. ohmeri, K. laetipori, $K$. nitidulidarum, $K$. transpacifica, $K$. meredithae have been described [33-35].

\section{Attributes pertaining xylose metabolism}

Xylose reductase and xylitol dehydrogenase enzyme activities pertaining to xylose metabolism [36, 37] were exhibited by both the strains but levels were low. The activities suggested the presence of xylose metabolizing pathway in these strains but levels were too low and their ratio predicted the flow of the pathway towards ethanol production. Specific activities $\left(\mathrm{U} \mathrm{mg}^{-1}\right.$ protein) of the strains were found to be $0.024,0.2$ (XR) for strain 5 and 6 respectively, while 0.011 and $0.015(\mathrm{XDH})$ for strain 5 and strain 6 respectively.

\section{Fermentation and co-fermentation capabilities and effect of supplementation}

As evident from absorbance at $660 \mathrm{~nm}$, both the strains grew well on minimal medium with xylose as sole carbon source and also on mixture of xylose and glucose and fermented them to ethanol (data not shown). On minimal medium containing salts, ethanol was produced by both

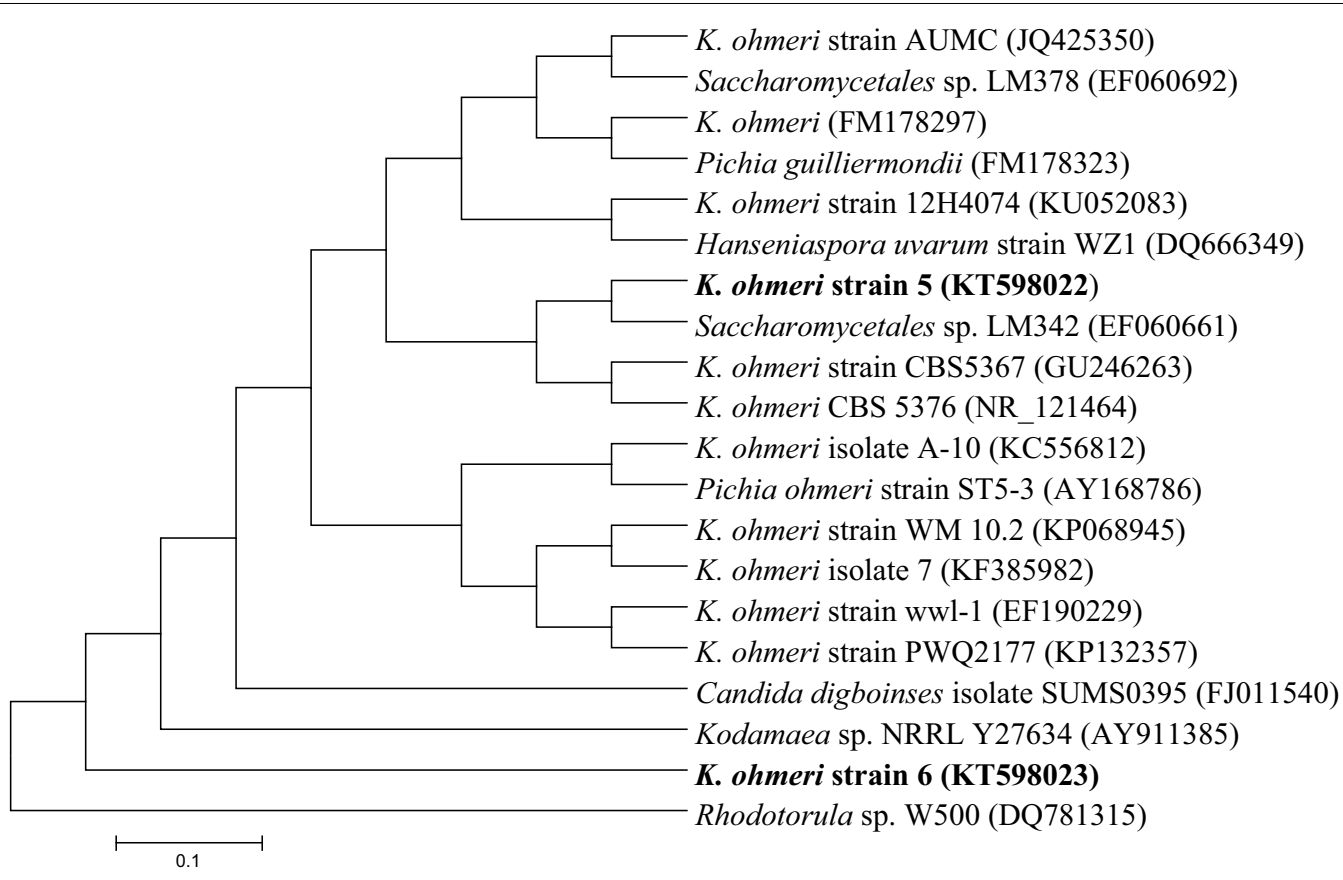

Fig. 2 Phylogenetic tree of K. ohmeri strain 5 and strain 6 
strains with fermentation efficiency of $\sim 25$ and $\sim 5 \%$ on glucose and xylose respectively (Fig. 3; Additional file 1: Table S2). Ethanol was the major product of glucose and xylose fermentation though trace amounts of xylitol and acetic acid were also detected during mixed sugar fermentation. Higher ethanol yield of $0.31 \mathrm{~g} \mathrm{~g}^{-1}$ from glucose with fermentation efficiency of $61 \%$ was obtained when minimal medium $\mathrm{w}$ as supplemented with $1 \%$ yeast extract (YE) and 1\% peptone (Table 3) without salts. In a study, D-arabitol production was observed as main product from glucose as the carbon source on rich medium (with $1 \%$ YE and $1 \%$ peptone) by $K$. ohmeri, and only trace amounts ethanol were observed. Production levels of polyols as fermentation products, largely depend on factors like proper ratio of nitrogen, carbon sources in the medium, original habitat of the fermenting organism and growth conditions [23]. Presence of salts in growth medium influence physiology, hamper growth and distress fermentation efficiency in yeasts while medium with organic supplements augment ethanol fermentation efficiency.

Supplementation with $0.1 \%$ YE and $0.1 \%$ peptone enhanced fermentation efficiency (to 50\%) (Table 4). Further enhancing level of supplementation in medium with higher concentrations of YE/peptone did not increase fermentation efficiency significantly. Studies have suggested significant role of cultivation media components to provide favorable conditions for growth and product formation [10]. Xylose consumption was also enhanced to $\sim 40 \%$ during co-fermentation and highest ethanol yield was $0.25 \mathrm{~g} \mathrm{~g}^{-1}$ sugar consumed when Kodamaea were grown on $10 \%$ total mixed sugars (5\% glucose $+5 \%$ xylose).

Amongst most of the pentose utilizing yeasts only a few have been reported to produce ethanol as major product from pentose fermentation [38]. A mixed sugar fermenting yeast, Candida lignohabitans possessing remarkable capability to ferment both pentoses and

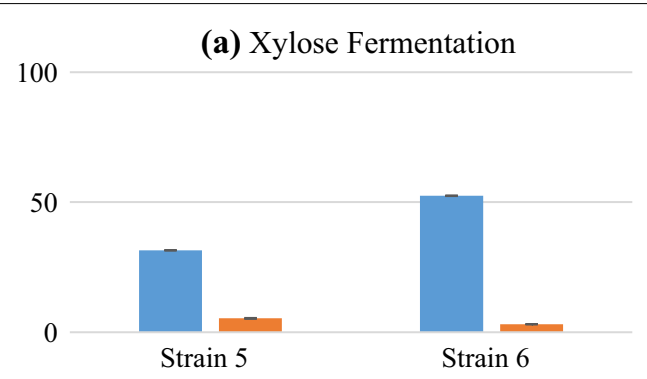

- Xylose consumed (\%) $\quad$ Fermentation efficiency $(\%)$

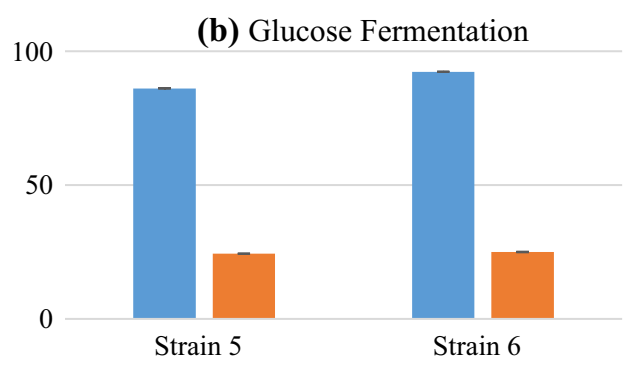

- Glucose consumed (\%) $\square$ Fermentation efficiency (\%)

Fig. 3 Xylose (a) and glucose (b) fermentation efficiency on minimal media with salts. Salts hamper the fermentation process as is visible from the lower fermentation efficiencies

\section{Table 3 Glucose utilization and ethanol yield of strain 5 and strain 6}

\begin{tabular}{|c|c|c|c|c|c|c|}
\hline \multirow{2}{*}{$\begin{array}{l}\text { Treatment } \\
\text { Strain } 5\end{array}$} & \multicolumn{3}{|c|}{ Glucose $\left(\mathrm{g} \mathrm{L}^{-1}\right)$} & \multicolumn{3}{|c|}{ Ethanol yield $\left(\mathbf{g ~ g}^{-1}\right)$} \\
\hline & & & & & & \\
\hline Time (h) & 96 & 108 & 120 & 96 & 108 & 120 \\
\hline $0.1 \%$ yeast extract $+0.1 \%$ peptone & $90.40 \pm 16.6$ & $97.95 \pm 1.8$ & $88.90 \pm 17.2$ & $0.16 \pm 0.04$ & $0.28 \pm 0.05$ & $0.20 \pm 0.09$ \\
\hline $0.5 \%$ yeast extract & $99.97 \pm 0.06$ & 100 & 100 & $0.16 \pm 0.09$ & $0.25 \pm 0.06$ & $0.28 \pm 0.12$ \\
\hline $1 \%$ yeast extract $+1 \%$ peptone & $97.74 \pm 3.71$ & $99.91 \pm 0.16$ & 100 & $0.13 \pm 0.02$ & $0.12 \pm 0.02$ & $0.12 \pm 0.02$ \\
\hline \multicolumn{7}{|l|}{ Strain 6} \\
\hline $0.1 \%$ yeast extract $+0.1 \%$ peptone & 100 & $99.9 \pm 0.17$ & 100 & 0.12 & $0.14 \pm 0.01$ & $0.12 \pm 0.02$ \\
\hline $0.5 \%$ yeast extract & $99.18 \pm 1.42$ & 100 & 100 & $0.22 \pm 0.05$ & $0.24 \pm 0.12$ & 0.13 \\
\hline $1 \%$ yeast extract $+1 \%$ peptone & 100 & $99.83 \pm 0.21$ & $100 \pm 0.01$ & $0.24 \pm 0.14$ & $0.31 \pm 0.10$ & $0.20 \pm 0.10$ \\
\hline $\mathrm{SE}_{\mathrm{m}}( \pm)$ & 1.61 & 0.23 & 1.71 & 0.02 & 0.02 & 0.02 \\
\hline CD @5\% & 4.46 & 0.64 & 4.73 & 0.05 & 0.06 & 0.05 \\
\hline
\end{tabular}

Ethanol yield $\left(\mathrm{g} \mathrm{g}^{-1}\right)=$ concentration of ethanol produced $\left(\mathrm{g} \mathrm{L}^{-1}\right) /$ concentration of sugar consumed $\left(\mathrm{g} \mathrm{L}^{-1}\right)$ \}

$S E_{m}$ standard error of mean, $C D$ critical difference 


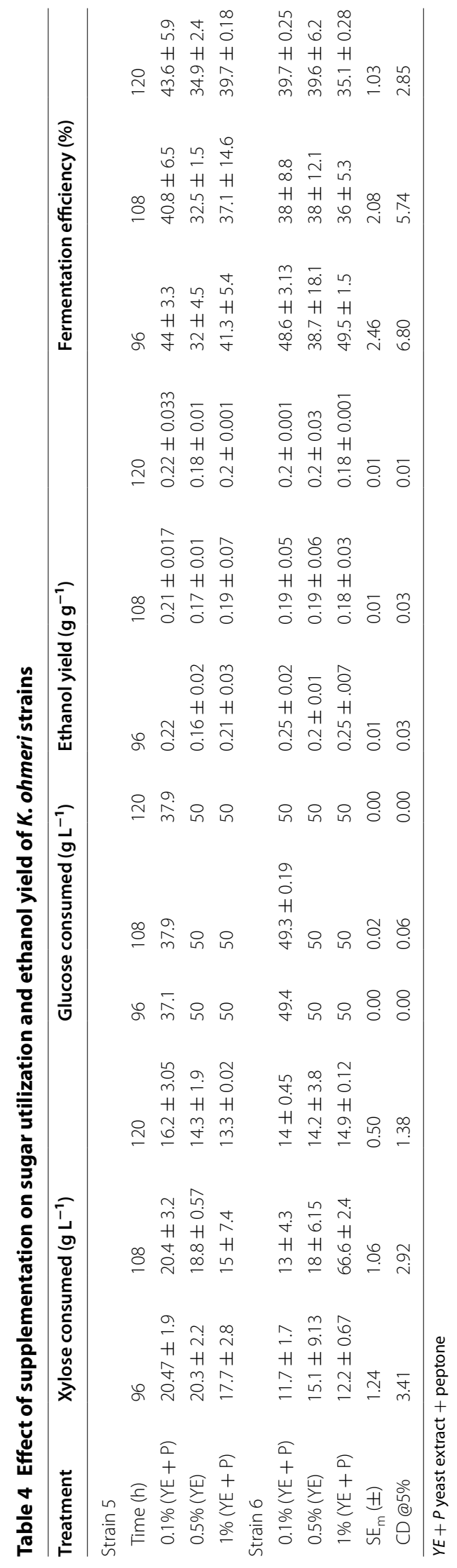


Table 5 Ethanol yields of pentose fermenting strains

\begin{tabular}{|c|c|c|c|}
\hline Strain & $\begin{array}{l}\text { Fermentable } \\
\text { sugar }\end{array}$ & $\begin{array}{l}\text { Ethanol yields } \\
\left(\mathbf{g ~ g}^{-1}\right)\end{array}$ & Reference \\
\hline C. lignohabitans & Glucose + xylose & 0.2 & {$[7]$} \\
\hline K. kakuduensis & Glucose & Traces & {$[21]$} \\
\hline K. ohmeri & Glucose & Traces (by product) & {$[23]$} \\
\hline K. ohmeri strain 5 & Xylose + glucose & 0.28 & This study \\
\hline K. ohmeri strain 6 & Xylose + glucose & 0.31 & This study \\
\hline
\end{tabular}

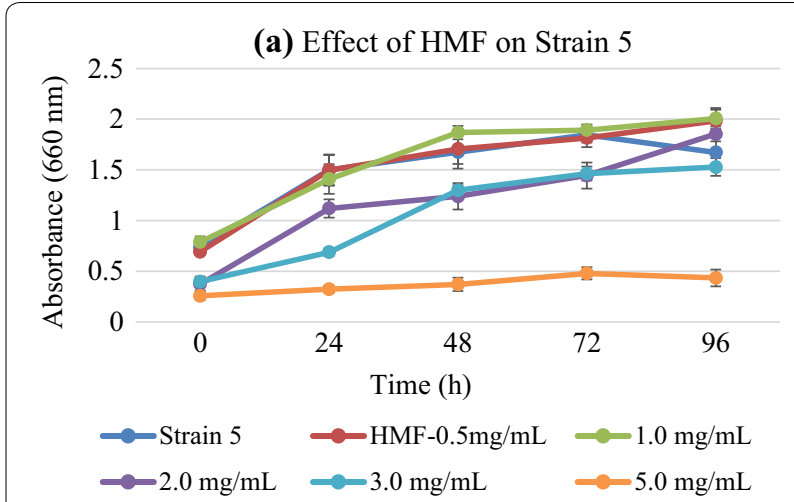

(b) Effect of HMF on Strain 6

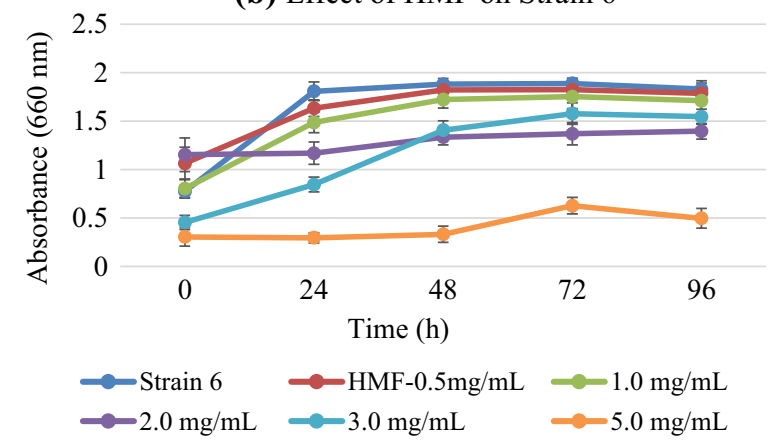

Fig. 4 Effect of hydroxy methyl furfural on strain 5 (a) and strain 6 (b). Growth pattern is similar to the control in case of strain 6 and $0.5-$ $3.0 \mathrm{~g} \mathrm{~L}^{-1}$ concentration of the HMF is not inhibitory for the growth

hexoses, exhibited highest ethanol yield of $0.2 \mathrm{~g} \mathrm{~g}^{-1}$ on rich medium containing $1 \%$ yeast extract and $2 \%$ soya peptone with $2-5 \%$ carbon sources, while no ethanol was detected on minimal medium without supplementation. This might be due to the lower biomass accumulation on minimal medium [7]. In this study, K. ohmeri strain 6 exhibited high ethanol yield during mixed substrate fermentation with minimal supplementation. Insignificant increase in fermentation efficiency upon medium with higher supplementation suggested to avoid excessive nutrient supplementation as it favors biomass production [23]. Table 5 shows ethanol yields of related yeast

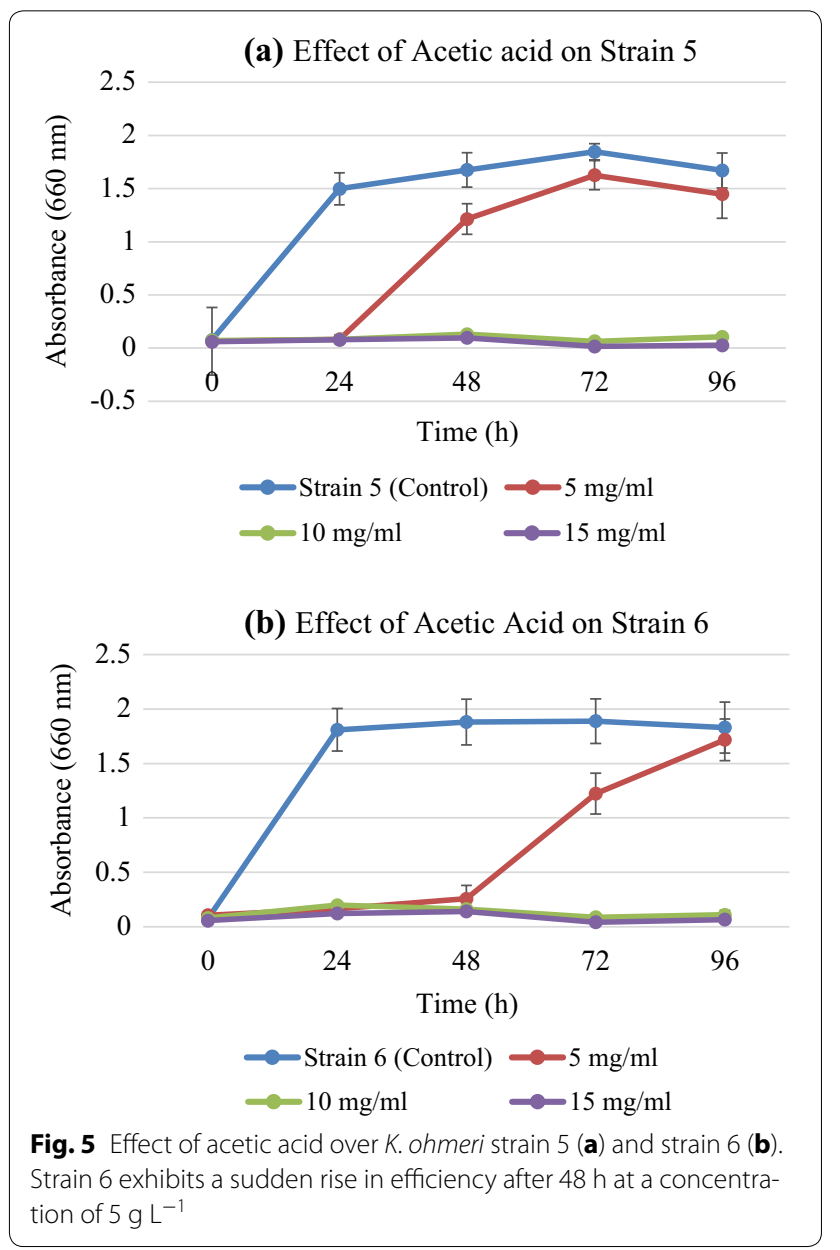

strains. Zheng et al. [3] observed stimulating effect of supplementation on acetone-butanol fermentation using Clostridium saccharoperbutylacetonicum and stated that lower supplementation is cost effective and reduces overall production cost.

\section{Inhibitor tolerance}

Lignocellulosic biomass is pretreated to facilitate higher conversion of biomass polysaccharides to fermentable sugars such as glucose, xylose, arabinose etc. This process generates by-products which inhibit growth of microbes and obstruct fermentation process. In general, these inhibitors are classified into four groups including lignin degradation by-products (phenolics), sugar degradation by-products (HMF and furfural), and products derived from the structure of the biomass and heavy metal ions (chromium and nickel) [39]. Effect of most commonly found inhibitors like HMF, furfural, acetic acid and formic acid was determined on growth of $K$. ohmeri strains.

Concentration ranges were selected based on yields commonly reported in literature and mostly encountered 

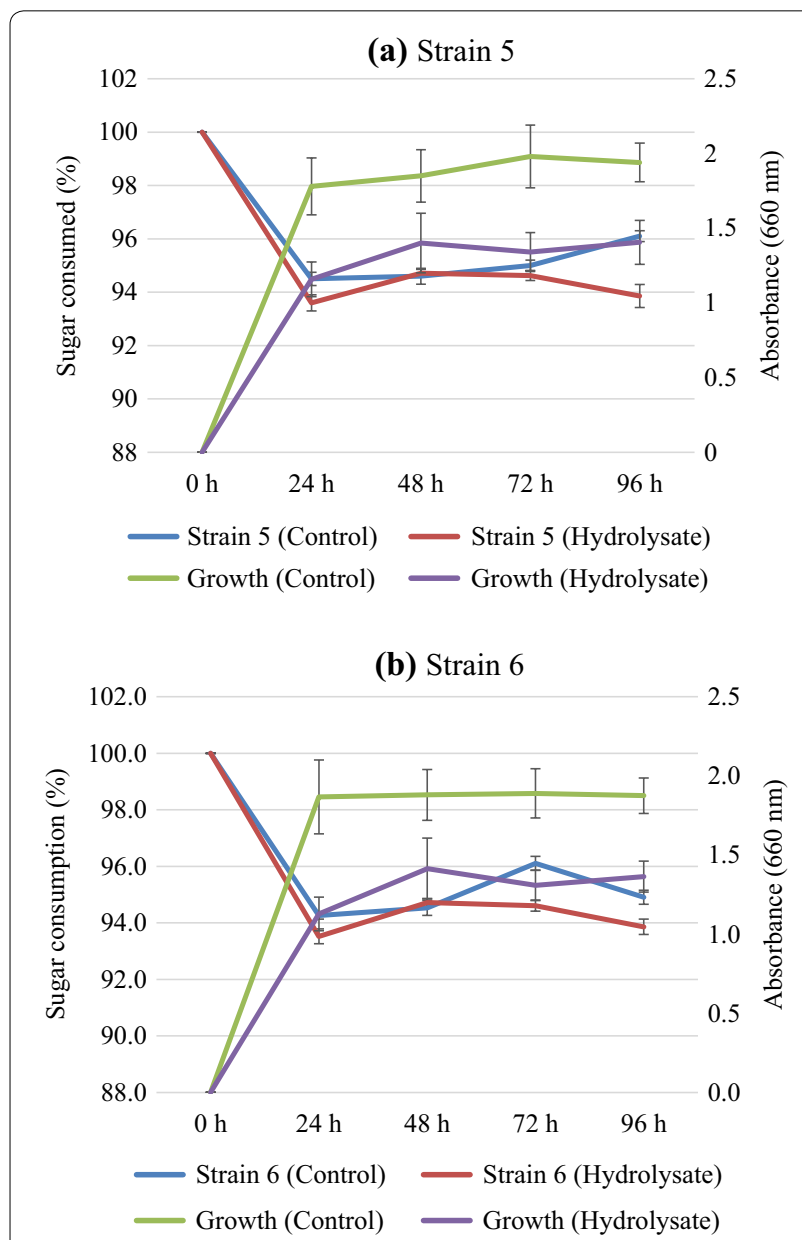

Fig. 6 Sugar consumption (\%) and growth of K. ohmeri strain 5 (a) and strain 6 (b) on biologically pretreated rice straw hydrolysate

in biomass hydrolysates after different pretreatments $[40,41]$. Increasing concentrations of HMF and furfural reduced growth of both strains as compared to controls (Fig. 4). Furfural was inhibitory in initial growth stages but inhibition was gradually overcome upon prolonged growth after $96 \mathrm{~h}$ (Additional file 1: Figure S3). This coincided with earlier observations that furfural can reduce growth rate above a certain concentration. It has been proved that furfural inhibits alcohol dehydrogenase (ADH) formation which lead to the accumulation of acetaldehyde intracellularly, causing enhanced lag phase of growth during which enzymes and co-enzymes are produced for the reduction of furfural [42]. HMF also posed similar threats on growth and ethanol productivity of $K$. ohmeri strains as growth was reduced and lesser biomass resulted in lesser ethanol production. K. ohmeri strains were found to be tolerant to HMF up to $3 \mathrm{~g} \mathrm{~L}^{-1}$ concentration while at $5 \mathrm{~g} \mathrm{~L}^{-1}$ concentration, growth was reduced.
Effect of organic acids on growth of $K$. ohmeri strains was more pronounced. With formic acid (3-11 $\left.\mathrm{g} \mathrm{L}^{-1}\right)$ and acetic acid (5-15 $\mathrm{g} \mathrm{L}^{-1}$ ), growth was highly affected due to $\mathrm{pH}$ change, as optimum $\mathrm{pH}$ for yeast growth is 5-6. Formic and acetic acids at concentration used in these experiments reduced $\mathrm{pH}$ to 3 leading to reduction in biomass production. In case of acetic acid, there was a sudden rise in growth of both strain 5 and strain 6 after 48 and $72 \mathrm{~h}$ respectively. Acetic acid at concentrations up to $6 \mathrm{~g} \mathrm{~L}^{-1}$ did not cause any reduction in growth of the strains [40] (Fig. 5). Acetic acid works by lowering intracellular $\mathrm{pH}$, which is neutralized by plasma membrane's ATPase by pumping out protons from the cell, thereby, leading to the production of additional ATPs by increasing ethanol production under anaerobic conditions due to enhanced biomass formation. This might be the reason for sudden rise in growth after a certain period as observed in case of $K$. ohmeri strains. Effect of formic acid was more severe and growth of both strains was impeded. Major cause of decreased growth was assumed to be lowering of $\mathrm{pH}$ as inhibitory effect of formic acid was nullified when $\mathrm{pH}$ was adjusted to optimum (data not shown). This reduction was due to drop in extracellular $\mathrm{pH}$ which causes diffusion of undissociated acids inside the cell leading to reduction in intracellular $\mathrm{pH}$ [43]. ABE fermentation was repressed by the production of acetic acid produced as a byproduct when $C$. saccharoperbutylacetonicum was grown on eucalyptus hydrolysates [3].

\section{Growth and ethanol production by $K$. ohmeri strains from biomass hydrolysates}

Kodamaea ohmeri strains were evaluated for growth and ethanol production on biomass hydrolysates prepared from biologically pretreated rice straw. Total sugar content in the hydrolysates was $\sim 1.3 \%$ (with $2 \%$ glucan loading and 57\% saccharification efficiency). Growth on hydrolysates was comparable to the control (Fig. 6). Maximum sugar consumption and ethanol production occurred within $24 \mathrm{~h}$. HPLC analyses of samples showed ethanol production and maximum ethanol level at $72 \mathrm{~h}$ by both the strains and it was $\sim 2$ and $1.3 \mathrm{~g} \mathrm{~L}^{-1}$ by strain 5 and strain 6 respectively (Table 6). Thus, these strains of $K$. ohmeri were able to grow and produce ethanol from paddy straw hydrolysates.

\section{Conclusions}

Screening for microbes capable of co-fermentation is necessary for efficient conversion of lignocellulosic biomass into ethanol with enhanced productivity. There is a significant advancement in developing a robust microbial strain with co-fermentation potential as well as tolerance to inhibitors. K. ohmeri strains, studied here showed promising mixed sugar fermentation potential 
Table 6 Ethanol yields of $K$. ohmeri strains from rice straw biomass hydrolysates

\begin{tabular}{lcccc}
\hline Ethanol produced $\left(\mathbf{g ~ L}^{\mathbf{- 1}}\right)$ & $\mathbf{2 4} \mathbf{h}$ & $\mathbf{4 8} \mathbf{h}$ & $\mathbf{7 2} \mathbf{h}$ & $\mathbf{9 6} \mathbf{h}$ \\
\hline K. ohmeri strain 5 (control) & $0.98 \pm 0.01$ & $0.065 \pm 0.003$ & $0.059 \pm 0.0005$ & $0.015 \pm 0.0025$ \\
K. ohmeri strain 5 (hydrolysate) & $0.3 \pm 0.001$ & $0.35 \pm 0.01$ & $1.92 \pm 0.04$ & $0.001 \pm 0.0015$ \\
K. ohmeri strain 6 (control) & $1.07 \pm 0.01$ & $1.15 \pm 0.05$ & $0.71 \pm 0.02$ & $0.26 \pm 0.02$ \\
K. ohmeri strain 6 (hydrolysate) & $0.12 \pm 0.03$ & $0.78 \pm 0.02$ & $1.28 \pm 0.01$ & $0.04 \pm 0.008$ \\
\hline
\end{tabular}

with enhanced xylose utilization. Strains were also tolerant to HMF, furfural, formic acid and could grow well in presence of acetic acid on prolonged incubation. The study emphasizes that this genus could provide robust native yeast strains with co-fermentation properties which can be evolved further. Lignocellulosic hydrolysates often generate unexpected results due to the presence of inhibitors, as they vary widely in nature [12]. These strains displayed efficient growth and ethanol production from biologically pretreated rice straw hydrolysates.

\section{Additional file}

Additional file 1. Table S1. Sugar utilization by K. ohmeri strain 5 and strain 6 . Table S2. Ethanol production, sugar consumption and fermentation efficiency of $K$. ohmeri strain 5 and strain 6 during xylose fermentation. Figure S1. Growth of $K$. ohmeri strain 5 and strain 6 on minimal medium with xylose as sole $C$ source. Figure S2. $K$. ohmeri strain $5(\mathbf{A})$ and strain $6(\mathbf{B})$ as observed under phase contrast microscope. Figure $\mathbf{S 3}$. Effect of furfural on K. ohmeri strain 5 (A) and strain 6 (B).

\section{Authors' contributions}

SS and PS carried out the experimental work. AA conceptualized the study, designing experiments and helped in the finalization of manuscript. Dr. SS performed HPLC of all the samples. Dr. LN and Dr. DP contributed for the saccharification and fermentation experimental work. All authors read and approved the final manuscript.

\section{Author details}

${ }^{1}$ Division of Microbiology, ICAR-Indian Agricultural Research Institute, New Delhi 110012, India. ${ }^{2}$ Amity Institute of Biotechnology, Amity University, Noida, U.P., India.

\section{Acknowledgements}

This work was supported by AMAAS (Grant No. 12-124), ICAR, India. Scanning electron microscopy was carried out in the Division of Entomology, ICAR-IARI, India.

\section{Competing interests}

The authors declare that they have no competing interests.

\section{Ethics approval and consent to participate}

Not applicable.

\section{Publisher's Note}

Springer Nature remains neutral with regard to jurisdictional claims in published maps and institutional affiliations.

Received: 22 December 2016 Accepted: 20 January 2018

Published online: 05 February 2018

\section{References}

1. Srirangan K, Akawi L, Moo-Young M, Chou CP (2012) Towards sustainable production of clean energy carriers from biomass resources. Appl Energy 100:172-186

2. Marcuschamer DK, Popiel PO, Simmons BA, Blanch HW (2010) Technoeconomic analysis of biofuels: a wiki-based platform for lignocellulosic biorefineries. Biomass Bioenergy 34:1914-1921

3. Zheng J, Tashiro Y, Wang Q, Sakai K, Sonomoto K (2015) Feasibility of acetone-butanol-ethanol fermentation from eucalyptus hydrolysate without nutrients supplementation. Appl Energy 140:113-119

4. Gong CS, Chen LF, Flickinger MC, Chiang LC, Tsao GT (1981) Production of ethanol from D-xylose by using D-xylose isomerase and yeasts. Appl Environ Microbiol 41:430-436

5. Fernandes S, Murray P (2010) Metabolic engineering for improved microbial pentose fermentation. Bioeng Bugs 1:424-428

6. Nilsson A, Gorwa-Grauslund MF, Hahn-Hägerdal B, Lidén G (2005) Cofactor dependence in furan reduction by Saccharomyces cerevisiae in fermentation of acid-hydrolyzed lignocellulose. Appl Environ Microbiol 71:7866-7871

7. Bellasio M, Mattanovich D, Sauer M, Marx H (2015) Organic acids from lignocellulose: Candida lignohabitans as a new microbial cell factory. J Ind Microbiol Biotechnol 42:681-691

8. Sharma S, Sharma S, Singh S, Arora A (2016) Improving yeast strains for pentose hexose co-fermentation: successes and hurdles. In Proceedings of the first international conference on recent advances in bioenergy research, Springer, Berlin, pp 23-41

9. Feng Y, Qi X, HI Jian, Sun RC, Jiang JX (2012) Effect of inhibitors on enzymatic hydrolysis and simultaneous saccharification fermentation for lactic acid production from steam explosion pretreated lespedeza stalks. BioResources 7:3755-3766

10. Hahn-Hägerdal B, Karhumaa K, Larsson CU, Gorwa-Grauslund M, Görgens J, van Zyl WH (2005) Role of cultivation media in the development of yeast strains for large scale industrial use. Microb Cell Fact 4:1-16

11. Casey E, Sedlak M, Ho NW, Mosier NS (2010) Effect of acetic acid and pH on the co-fermentation of glucose and xylose to ethanol by a genetically engineered strain of Saccharomyces cerevisiae. FEMS Yeast Res 10:385-393

12. Cortez D, Roberto I (2006) Effect of phosphate buffer concentration on the batch xylitol production by Candida guilliermondii. Lett Appl Microbiol 42:321-325

13. Jeffries TW (1981) Conversion of xylose to ethanol under aerobic conditions by Candida tropicalis. Biotechnol Lett 3:213-218

14. Karczewska H (1958) Some observations on pentose utilization by Candida tropicalis. Comptes-rendus des travaux du Laboratoire Carlsberg 31:251-258

15. De Bari I, De Canio P, Cuna D, Liuzzi F, Capece A, Romano P (2013) Bioethanol production from mixed sugars by Scheffersomyces stipitis free and immobilized cells, and co-cultures with Saccharomyces cerevisiae. New Biotechnol 30:591-597

16. Bhadra B, Rao RS, Singh PK, Sarkar PK, Shivaji S (2008) Yeasts and yeast-like fungi associated with tree bark: diversity and identification of yeasts producing extracellular endoxylanases. Curr Microbiol 56:489-494

17. Sreenath $H$, Jeffries $T$ (2000) Production of ethanol from wood hydrolyzate by yeasts. Bioresour Technol 72:253-260

18. Rao RS, Bhadra B, Shivaji S (2008) Isolation and characterization of ethanol-producing yeasts from fruits and tree barks. Lett Appl Microbiol 47:19-24

19. Long TM, Su YK, Headman J, Higbee A, Willis LB, Jeffries TW (2012) Cofermentation of glucose, xylose, and cellobiose by the beetle-associated yeast Spathaspora passalidarum. Appl Environ Microbiol 78:5492-5500 
20. Nogué VS, Karhumaa K (2014) Xylose fermentation as a challenge for commercialization of lignocellulosic fuels and chemicals. Biotechnol Lett 37:761-772

21. Lachance MA, Bowles JM, Starmer WT, Barker JS (1999) Kodamaea kakaduensis and Candida tolerans, two new ascomycetous yeast species from Australian Hibiscus flowers. Can J Microbiol 45:172-177

22. Daniel HM, Vrancken G, Takrama JF, Camu N, De Vos P, De Vuyst L (2009) Yeast diversity of Ghanaian cocoa bean heap fermentations. FEMS Yeast Res 9:774-783

23. Zhu HY, Xu H, Dai XY, Zhang Y, Ying HJ, Ouyang PK (2010) Production of D-arabitol by a newly isolated Kodamaea ohmeri. Bioprocess Biosyst Eng 33:565-571

24. Altschul SF, Madden TL, Schaffer AA, Zhang J, Zhang Z, Miller W, Lipman DJ (1997) Gapped BLAST and PSI-BLAST: a new generation of protein database search programs. Nucleic Acids Res 25:3389-3402

25. Qi X, Luo Y, Wang X, Zhu J, Lin J, Zhang H, Chen F, Sun W (2015) Enhanced D-arabitol production by Zygosaccharomyces rouxii JM-C46: isolation of strains and process of repeated-batch fermentation. J Ind Microbiol Biotechnol 42:807-812

26. McMillan J (1993) Xylose fermentation to ethanol. A review. National Renewable Energy Lab, Golden

27. Updegraff DM (1969) Semi micro determination of cellulose in biological materials. Anal Biochem 32:420-424

28. Mohanram S, Rajan K, Carrier DJ, Nain L, Arora A (2015) Insights into biological delignification of rice straw by Trametes hirsuta and Myrothecium roridum and comparison of saccharification yields with dilute acid pretreatment. Biomass Bioenergy 76:54-60

29. Miller GL (1959) Use of dinitrosalicylic acid reagent for determination of reducing sugar. Anal Chem 31:426-428

30. Arora A, Priya S, Sharma P, Sharma S, Nain L (2016) Evaluating biological pretreatment as a feasible methodology for ethanol production from paddy straw. Biocatal Agric Biotechnol. https://doi.org/10.1016/j. bcab.2016.08.006

31. Tyagi N, Madan H, Pathak S (2014) Phytochemical screening and estimation of total phenolics and total flavonoid content of Lagenaria siceraria Praecitrullus fistulosus (50:50) fruit and their mixture. IJPRS 3(2):882-890

32. Kimura M (1980) A simple method for estimating evolutionary rate of base substitutions through comparative studies of nucleotide sequences. J Mol Evol 16:111-120
33. Freitas LFD, Barriga EJC, Barahona PP, Lachance MA, Rosa CA (2013) Kodamaea transpacifica f.a., sp. nov., a yeast species isolated from ephemeral flowers and insects in the Galápagos Islands and Malaysia: further evidence for ancient human transpacific contacts. Int J Syst Evol Microbiol 63:4324-4329

34. Rosa CA, Lachance MA, Starmer WT, Barker JSF, Bowles JM, Schlag-Edler B (1999) Kodamaea nitidulidarum, Candida restingae and Kodamaea anthophila, three new related yeast species from ephemeral flowers. Int J Syst Bacteriol 49:309-318

35. Sylvester K, Wang Q, James B, Mendez R, Hulfachor A, Hittinger C (2015) Temperature and host preferences drive the diversification of Saccharomyces and other yeasts: a survey and the discovery of eight new yeast species. FEMS Yeast Res 15(3):fov002

36. Yokoyama SI, Suzuki T, Kawai K, Horitsu H, Takamizawa K (1995) Purification, characterization and structure analysis of NADPHdependent D-xylose reductase from Candida tropicalis. J Ferment Bioeng 79(3):217-223

37. Ikeuchi I, Kiritani R, Azuma M, Ooshima H (2000) Effect of D-glucose on induction of xylose reductase and xylitol dehydrogenase in Candida tropicalis in the presence of $\mathrm{NaCl}$. J Basic Microbiol 40(3):167-175

38. Hahn-Hagerdal B, Karhumaa K, Fonseca C, Spencer-Martins I, GorwaGrauslund MF (2007) Towards industrial pentose-fermenting yeast strains. Appl Microbiol Biotechnol 74:937-953

39. Parajó J, Domínguez H, Domínguez J (1996) Charcoal adsorption of wood hydrolysates for improving their fermentability: influence of the operational conditions. Bioresour Technol 57:179-185

40. van der Pol EC, Bakker RR, Baets P, Eggink G (2014) By-products resulting from lignocellulose pretreatment and their inhibitory effect on fermentations for (bio) chemicals and fuels. Appl Microbiol Biotechnol 98:9579-9593

41. Wikandari R, Millati R, Syamsiyah S, Muriana R, Ayuningsih Y (2010) Effect of furfural, hydroxyl methyl furfural and acetic acid on indigeneous microbial isolate for bioethanol production. Agric J 5:105-109

42. Banerjee N, Bhatnagar R, Viswanathan L (1981) Inhibition of glycolysis by furfural in Saccharomyces cerevisiae. Eur J Appl Microbiol Biotechnol 11:226-228

43. Orij R, Brul S, Smits GJ (2011) Intracellular pH is a tightly controlled signal in yeast. Biochimica Biophysica Acta 1810:933-944

\section{Submit your manuscript to a SpringerOpen ${ }^{\circ}$ journal and benefit from:}

- Convenient online submission

- Rigorous peer review

- Open access: articles freely available online

- High visibility within the field

Retaining the copyright to your article

Submit your next manuscript at springeropen.com 